\title{
Reduced Serum Phosphorus Levels Were Associated with Metabolic Syndrome in Men But Not in Women: A Cross-Sectional Study among the Japanese Population
}

\author{
Masanori Shimodaira, ${ }^{a, b}$ Shinji Okaniwa ${ }^{a}$ Tomohiro Nakayama $^{b}$ \\ ${ }^{a}$ Department of Internal Medicine, lida Municipal Hospital, Nagano, and b Division of Companion Diagnostics, \\ Department of Pathology of Microbiology, Nihon University School of Medicine, Tokyo, Japan
}

\section{Keywords}

Serum phosphorus · Metabolic syndrome $\cdot$ Risk factor .

Gender

\begin{abstract}
Background: Reduced serum phosphorus (SP) levels are reported to be associated with insulin resistance and metabolic syndrome (MetS). However, there have been a few gender-specific studies although SP levels are substantially different between men and women. Methods: This is a cross-sectional study. A total of 16,041 subjects $(9,076$ men and 6,965 women) were analyzed. The subjects were divided into 3 groups of gender-specific tertiles based on phosphorus levels: the lowest $\left(T_{1}\right)$, middle $\left(T_{2}\right)$, and the highest $\left(T_{3}\right)$. Results: SP levels were significantly lower in subjects with MetS than in those without MetS in men but not in women. Waist circumference and fasting plasma glucose were negatively and high-density lipoprotein cholesterol was positively correlated with SP levels both in men and women. Blood pressure (BP) and triglycerides (TG) were negatively correlated with SP levels in men, while they were positively correlated with SP levels in women. Lower SP levels were associated with the prevalence of MetS in men $\left(\mathrm{T}_{1} ; 19.9 \%, \mathrm{~T}_{2} ; 16.9 \%\right.$, and $\left.\mathrm{T}_{3} ; 14.3 \% ; p<0.001\right)$ but not in women $\left(\mathrm{T}_{1} ; 14.1 \%, \mathrm{~T}_{2}\right.$; $16.6 \%$, and $\mathrm{T}_{3} ; 15.3 \% ; p=0.282$ ). Conclusion: $\mathrm{BP}$ and $\mathrm{TG}$ were
\end{abstract}

unexpectedly positively correlated with SP levels in women. Reduced SP levels were associated with MetS in men but not in women.

(c) 2017 S. Karger AG, Basel

\section{Introduction}

Metabolic syndrome (MetS) is a constellation of metabolic disorders including abdominal obesity, atherogenic dyslipidemia (elevated triglyceride [TG] and/or reduced high-density lipoprotein cholesterol [HDL-C]), elevated blood pressure (BP), and high fasting plasma glucose (FPG) [1]. Phosphorus is an essential mineral required for biological activities, biochemical reactions, and energy metabolism [2]. Therefore, a phosphorus imbalance may contribute to the development of metabolic disorders $[3,4]$. Several studies have found that lower serum phosphorus (SP) levels are associated with impaired glucose tolerance and insulin resistance [5-7]. Furthermore, hypertensive subjects have significantly lower phosphorus levels compared with healthy individuals [8]. In contrast, higher levels of SP are thought to be associated with lower TG and higher HDL-C levels $[9,10]$. These studies indicate that a disturbance in phosphorus metabolism may represent a key feature of MetS and its components.

\section{KARGER}

(c) 2017 S. Karger AG, Basel

E-Mail karger@karger.com

www.karger.com/anm
Masanori Shimodaira, $\mathrm{MD}, \mathrm{PhD}$

Department of Internal Medicine, Iida Municipal Hospital

438 Yawata-machi, Iida

Nagano 395-8502 (Japan)

E-Mail masanori19810813@yahoo.co.jp 
The prevalence of MetS and its phenotypes differs between men and women [11-13]. For example, in the Japanese population, the prevalence of MetS with elevated FPG levels is higher in men than in women [11]. Furthermore, while SP levels differ by gender, there have been a few gender-stratified studies investigating the relationship between SP levels and MetS and its components [14]. Therefore, we conducted the present gender-stratified cross-sectional study to investigate the relationship between SP and metabolic parameters in the Japanese population.

\section{Materials and Methods}

\section{Study Subjects}

This study included individuals undergoing routine health checkups at the Iida Municipal Hospital from January 2008 to December 2015. When individuals had data from multiple visits, we included only the data acquired during the first visit. Out of 16,735 individuals, we excluded individuals with a possibility of advanced renal dysfunction, such as those with an estimated glomerular filtration rate $(\mathrm{eGFR})<30 \mathrm{~mL} / \mathrm{min} / 1.73 \mathrm{~m}^{2}$, and evidence of albuminuria indicated by urine dipstick albumin $>2+$. In addition, we excluded individuals of advanced age ( $>80$ years). Consequently, we included a total of 16,041 subjects (men, $n=9,076$ and women, $n=6,965)$ in the present analysis. The study was approved by the Institution Ethics Review Board of Iida Municipal Hospital in agreement with the Helsinki Declaration of 1975. Informed consent was obtained from all participants.

\section{Definition of the Variables}

According to the National Cholesterol Education Program, Adult Treatment Panel III criteria [1], MetS was defined by the fulfillment of 3 or more components of the 5 following abnormalities: (1) waist circumference (WC) $\geq 90$ and $\geq 80 \mathrm{~cm}$ in Japanese men and women, respectively; (2) systolic BP $\geq 130 \mathrm{~mm} \mathrm{Hg}$ and/or diastolic $\mathrm{BP} \geq 85 \mathrm{~mm} \mathrm{Hg}$, or treatment for previously diagnosed hypertension; (3) $\mathrm{TG} \geq 150 \mathrm{mg} / \mathrm{dL}$ or receiving specific treatment for hypertriglycemia; (4) HDL-C $<40 \mathrm{mg} / \mathrm{dL}$ in men and $<50 \mathrm{mg}$ / day in women, or receiving specific treatment for low HDL-C; and (5) FPG $\geq 100 \mathrm{mg} / \mathrm{dL}$ or previously diagnosed diabetes. The individuals with fewer than 3 criteria for the diagnosis of MetS served as the controls. SP levels were categorized by tertiles with tertile 1 $\left(\mathrm{T}_{1}\right)$ representing the lowest phosphorus levels; $\mathrm{T}_{2}$ : meddle phosphorus levels; and $\mathrm{T}_{3}$ : the highest phosphorus levels.

\section{Clinical and Anthropometric Measurements}

Height and weight were measured with the subjects wearing light clothing and no shoes using a fixed stadiometer and a digital scale, which was used to calculate the body mass index (BMI). WC was measured between the lower rib margin and the iliac crest after a normal expiratory breath. Systolic and diastolic BP was measured using an automated measuring device (HEM-7080IT, Omron Co., Tokyo, Japan). Two separate BP measurements were taken after 15 min of rest, with at least a 30-s interval between the 2, and the mean of these was considered to be the participant's BP.

Phosphorus Levels and Metabolic Syndrome
Information regarding various comorbid conditions, including hypertension, dyslipidemia, and diabetes was collected by conducting in-person interviews. The smoking status and alcohol consumption of the study participants were assessed using a standardized questionnaire. Smoking status was categorized as either current smokers (daily and occasional smoking) or non-smokers (never and former smoking). Alcohol consumption was classified as current drinkers (at least once per week) and non-current drinkers.

\section{Laboratory Measurements}

Blood samples were obtained after an overnight fast. The measurements of the total serum cholesterol, TG, HDL-C, low density lipoprotein cholesterol, uric acid, high sensitivity C-reactive protein (hs-CRP), and creatinine were determined using standard methods (Hitachi 47 automatic analyzer, Hitachi Ltd., Japan). eGFR was calculated using the formula of the Japanese Society of Nephrology according to the equation: $194 \times$ serum creatinine $\mathrm{e}^{-1.094} \times$ age $^{-0.287} \mathrm{~mL} / \mathrm{min} / 1.73 \mathrm{~m}^{2}$, which was further multiplied by 0.739 for female subjects [15]. FPG was measured using the hexokinase method (GLU-HK, Shinotest Inc., Japan). SP was measured by the enzymatic method with maltose phosphorylase (Kainos Laboratory Inc., Japan). The coefficient of variation for SP determination was less than 1.0 and $0.6 \%$ for low- and high-level quality control specimens, respectively.

\section{Statistical Analysis}

Statistical analyses were performed using the SPSS software version 21.0 (SPSS Inc. IL, USA). Gender-specific characteristics of the study population were analyzed using independent $t$ tests for continuous variables and the chi-square test for dichotomous variables. Skewed distribution variables were analyzed after a logarithmic transformation. The correlation between the SP levels and the variables of the MetS components was analyzed using Pearson's correlation analysis, and a partial correlation analysis was performed to control confounding factors (e.g., age, BMI, hs-CRP, eGFR, smoking and alcohol status, and the use of antihypertensive, antidiabetic, and hypolipidemic drugs). The differences in the mean values of the MetS components according to SP level tertiles were evaluated using an analysis of covariance. To determine the adjusted ORs for the incidence of MetS and its components between the tertile categories, a logistic regression analysis was used to adjust the confounding factors.

\section{Results}

\section{Characteristics of the Subjects with and without MetS}

The physical and biochemical characteristics of the study subjects are summarized in Table 1 . The prevalence of MetS in men and women were 17.0 and $15.4 \%$, respectively. Of the men, the subjects with MetS exhibited significantly lower SP levels than those without MetS (3.04 \pm 0.44 vs. $3.13 \pm 0.42 \mathrm{mg} / \mathrm{dL}$, respectively). Conversely, in women, there was no difference in SP levels between the 2 groups. 
Table 1. Demographic and metabolic characteristics of study participants

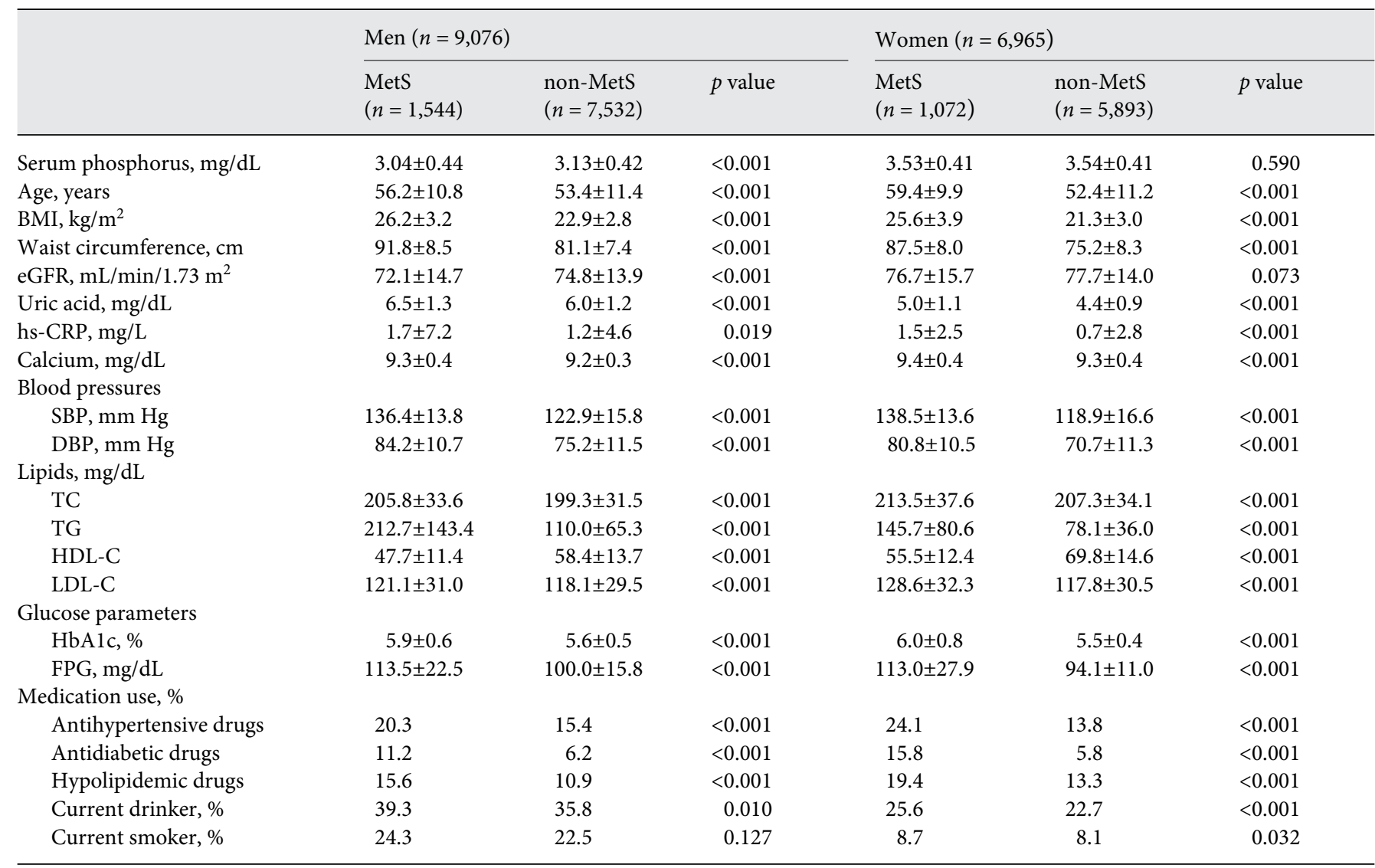

MetS, metabolic syndrome; BMI, body mass index; eGFR, estimated glomerular filtration rate; SBP, systolic blood pressure; DBP, diastolic blood pressure; hs-CRP, high sensitive C-reactive protein; TC, total cholesterol; TG, triglycerid; HDL-C, HDL-cholesterole; LDL-C, LDL-cholesterol; FPG, fasting plasma glucose.

\section{Correlations between Serum Phosphorus Levels and MetS Components}

In both genders, WC and FPG were negatively correlated and HDL-C was positively correlated with SP levels even after an adjustment for the confounding factors ( $\mathrm{Ta}$ ble 2). Interestingly, there were gender-related differences in the correlation between SP levels and systolic and diastolic BP and TG levels - a negative correlation among male subjects but a positive correlation among female subjects.

\section{Prevalence of MetS and Values of the MetS \\ Components According to Serum Phosphorus Level \\ Tertiles}

The prevalence of MetS and mean values of the MetS components adjusted for the confounding factors between the different SP level tertiles are shown in Table 3.
In men, the prevalence of MetS increased as SP levels decreased. However, in women, there was no difference in the prevalence of MetS between the 3 groups. In both genders, as SP levels decreased, WC and FPG levels increased and HDL-C levels decreased. On the other hand, gender differences were identified for the relationship between systolic and diastolic BP and TG levels and SP levels; there was a negative association among male subjects but a positive association among female subjects.

\section{ORs of the MetS and Its Components According to \\ Serum Phosphorus Level Tertiles}

The unadjusted and adjusted ORs for the presence of MetS and its components according to SP levels are presented in Table 4 . In both genders, the ORs of elevated FPG increased according to a decrease in SP levels. Only in men, the ORs of elevated WC, elevated TG, and 
Table 2. Correlation coefficients between serum phosphorus levels and values of MetS traits

\begin{tabular}{|c|c|c|c|c|c|c|c|c|}
\hline & \multicolumn{4}{|l|}{ Men } & \multicolumn{4}{|c|}{ Women } \\
\hline & \multicolumn{2}{|c|}{ unadjusted } & \multicolumn{2}{|c|}{ adjusted* } & \multicolumn{2}{|c|}{ unadjusted } & \multicolumn{2}{|c|}{ adjusted* } \\
\hline & $r$ & $p$ value & $r$ & $p$ value & $r$ & $p$ value & $r$ & $p$ value \\
\hline $\mathrm{WC}, \mathrm{cm}$ & -0.085 & $<0.001$ & -0.066 & $<0.001$ & -0.053 & $<0.001$ & -0.095 & $<0.001$ \\
\hline $\mathrm{SBP}, \mathrm{mm} \mathrm{Hg}$ & -0.110 & $<0.001$ & -0.096 & $<0.001$ & 0.031 & 0.025 & 0.055 & $<0.001$ \\
\hline $\mathrm{DBP}, \mathrm{mm} \mathrm{Hg}$ & -0.096 & $<0.001$ & -0.092 & $<0.001$ & 0.049 & $<0.001$ & 0.095 & $<0.001$ \\
\hline $\mathrm{TG}, \mathrm{mg} / \mathrm{dL}^{\dagger}$ & -0.019 & 0.011 & -0.025 & 0.012 & 0.049 & 0.001 & 0.047 & 0.002 \\
\hline HDL-C, mg/dL & 0.048 & $<0.001$ & 0.031 & 0.014 & 0.098 & $<0.001$ & 0.083 & $<0.001$ \\
\hline $\mathrm{FPG}, \mathrm{mg} / \mathrm{dL}$ & -0.054 & $<0.001$ & -0.052 & $<0.001$ & -0.037 & 0.008 & -0.102 & $<0.001$ \\
\hline
\end{tabular}

MetS, metabolic syndrome; WC, waist circumference; SBP, systolic blood pressure; DBP, diastolic blood pressure; TG, triglycerid; HDL-C, HDL-cholesterole; FPG, fasting plasma glucose.

${ }^{\dagger}$ Log-transformed

* Partial correlation analysis adjusted for age, BMI, high sensitivity C-reactive protein, estimated glemerular filtration rate, smoking and alcohol status, and use antihypertensive, antidiabetic, and hypolipidemic drugs.

Table 3. Prevalence of MetS and adjusted mean values of MetS components according to serum phosphorus level tertiles

\begin{tabular}{|c|c|c|c|c|c|c|c|c|}
\hline MetS, \% & 19.9 & 16.9 & 14.3 & $<0.001$ & 14.1 & 16.6 & 15.3 & 0.282 \\
\hline $\mathrm{WC}, \mathrm{cm}$ & $84.1 \pm 8.1$ & $83.0 \pm 9.0$ & $82.4 \pm 8.7$ & $<0.001$ & $77.6 \pm 9.6$ & $77.0 \pm 9.0$ & $76.8 \pm 9.1$ & 0.030 \\
\hline $\mathrm{TG}, \mathrm{mg} / \mathrm{dL}$ & $131.2 \pm 101.1$ & $127.7 \pm 95.5$ & $120.5 \pm 73.5$ & 0.002 & $82.6 \pm 40.9$ & $85.3 \pm 44.9$ & $86.4 \pm 48.5$ & 0.049 \\
\hline $\mathrm{HDL}-\mathrm{C}, \mathrm{mg} / \mathrm{dL}$ & $55.5 \pm 13.5$ & $57.1 \pm 13.7$ & $57.9 \pm 14.7$ & 0.004 & $66.5 \pm 13.7$ & $67.6 \pm 15.2$ & $68.3 \pm 16.1$ & 0.035 \\
\hline FPG, mg/dL & $103.4 \pm 18.5$ & $102.1 \pm 16.2$ & $101.6 \pm 18.6$ & 0.003 & $98.8 \pm 19.6$ & $96.5 \pm 14.3$ & $95.5 \pm 13.3$ & $<0.001$ \\
\hline
\end{tabular}

MetS, metabolic syndrome; WC, waist circumference; SBP, systolic blood pressure; DBP, diastolic blood pressure; TG, triglycerid; HDL-C, HDL-cholesterole; FPG, fasting plasma glucose.

Values of MetS components were adjusted for age, BMI, high sensitivity C-reactive protein, estimated glemerular filtration rate, smoking and alcohol status, and use of antihypertensive, antidiabetic, and hypolipidemic drugs.

reduced HDL-C increased in accordance with a decrease in SP levels. Gender differences were found in the association between the ORs of elevated BP and SP levels; a positive association was found in men, whereas there was a negative association in women. These relationships remained significant after adjusting for the confounding factors. In men, because of the significant association between the MetS components and SP levels, ORs of MetS decreased with SP levels. The adjusted ORs in $\mathrm{T}_{2}$ and $\mathrm{T}_{1}$ were 1.19 and 1.23 , respectively, using $\mathrm{T}_{3}$ as a reference.

Phosphorus Levels and Metabolic Syndrome

\section{Discussion}

This gender-stratified analysis conducted in a large general population that represents a single ethnicity demonstrated gender differences in the association between SP levels and the incidence of MetS; decreased phosphorus levels were related to an increased incidence of MetS only among male subjects. We also identified a negative association between systolic and diastolic BPs and TG among male subjects; however, an inverse association was observed among female subjects.

Ann Nutr Metab 2017;71:150-156 
Table 4. OR and $95 \% \mathrm{CI}$ for the prevalence of MetS and its components according to serum phosphorus level tertiles

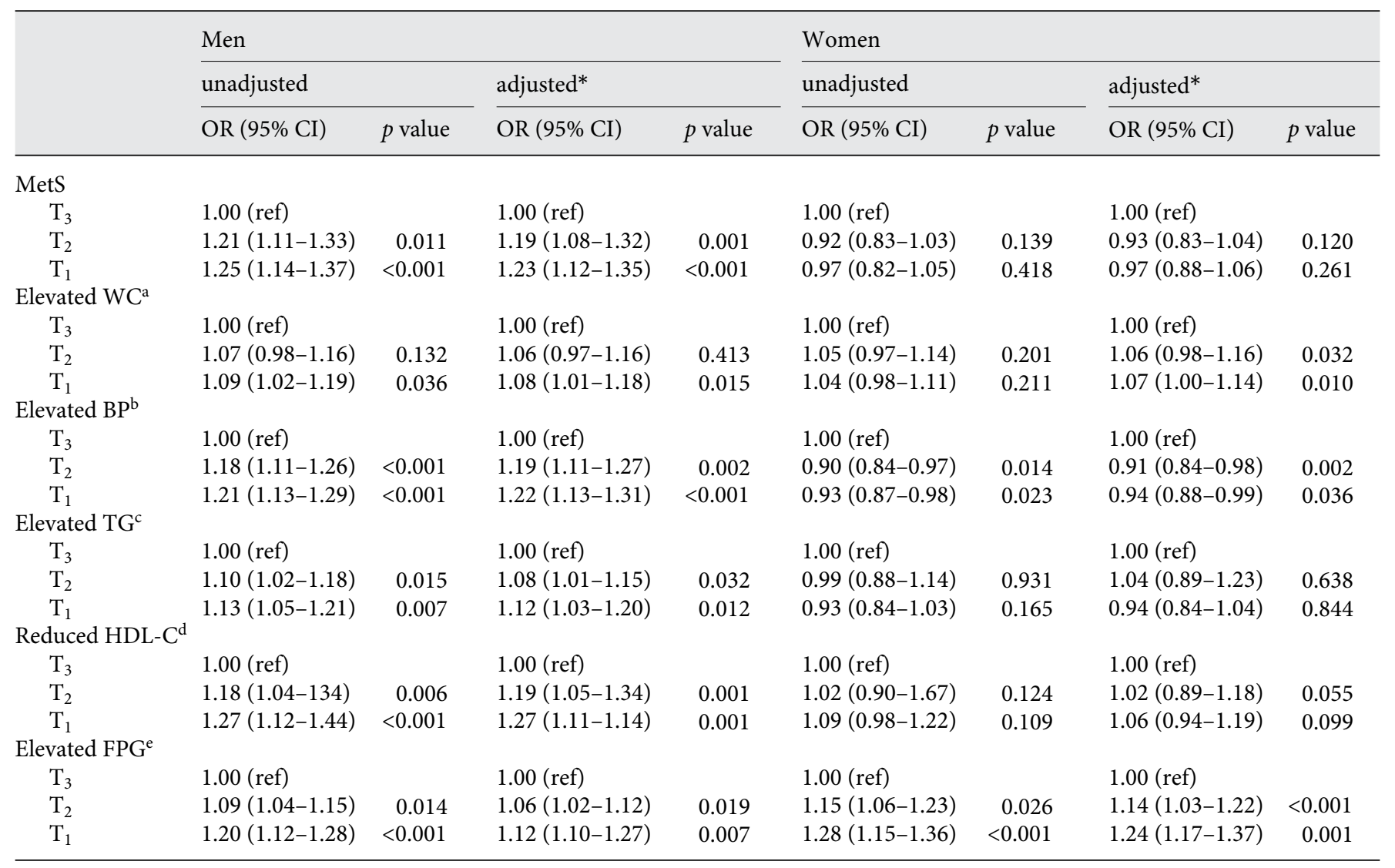

MetS, metabolic syndrome; WC, waist circumference; BP, blood pressure; TG, triglycerid; HDL-C, HDL-cholesterole; FPG, fasting plasma glucose.

* adjusted for age, BMI, high sensitivity C-reactive protein, estimated glemerular filtration rate, smoking and alcohol status, and use of antihypertensive, antidiabetic, and hypolipidemic drugs.

${ }^{\text {a }} \mathrm{WC} \geq 90 \mathrm{~cm}$ in men and $\geq 80 \mathrm{~cm}$ in women.

b systolic BP $\geq 130 \mathrm{~mm} \mathrm{Hg}$ and/or diastolic BP $\geq 85 \mathrm{~mm} \mathrm{Hg}$.

c $\mathrm{TG} \geq 150 \mathrm{mg} / \mathrm{dL}$.

${ }^{\mathrm{d}} \mathrm{HDL}-\mathrm{C}<40 \mathrm{mg} / \mathrm{dL}$ in men and $<50 \mathrm{mg} /$ day in women.

${ }^{\text {e }} \mathrm{FPG} \geq 100 \mathrm{mg} / \mathrm{dL}$

Elevated TG and reduced HDL-C levels have been referred to as atherogenic dyslipidemia and is thought to be a significant predictor of future high BP and diabetes [16]. This study demonstrated that the correlations of SP and TG were different between both genders. Onat et al. [16] have reported that CRP was independently related to MetS of atherogenic dyslipidemia in men but not in women. This suggests that atherogenic dyslipidemia in women with MetS was closely associated with inflammation/oxidative stress. Lipoprotein(a) is associated with inflammatory markers, while elevated TG is linked to high lipoprotein(a) in men and low lipoprotein(a) in women [17]. As shown in Table 1, the female subjects had lower hs-CRP levels than the male subjects. However the exact reason for this phenomenon is still unclear; the different associations of SP levels and TG between men and women may be attributable to the differences in the impact of chronic low-grade inflammation on lipid metabolism in MetS.

Several studies have reported a negative correlation between SP levels and BP [10]; however, the correlation remains inconclusive $[18,19]$. According to Haglin et al. [3], the correlation was not significant in women. In their study, the mean SP and BMI of women were 3.28 $\mathrm{mg} / \mathrm{dL}$ and $31.4 \mathrm{~kg} / \mathrm{m}^{2}$, respectively, whereas our study, they were $3.54 \mathrm{mg} / \mathrm{dL}$ and $22-23 \mathrm{~kg} / \mathrm{m}^{2}$, respectively. These differences in SP and/or BMI values may explain 
the differences regarding the association between SP levels and BP in the 2 populations. However, we could not identify a clear rationale for the gender-related differences concerning the association of SP levels with BP in this study.

In accordance with previous studies [10,7], we demonstrated that higher SP levels were associated with lower FPG levels in both genders. The relationship between SP and glucose is believed to be mediated by insulin, which increases the transfer of phosphate from extracellular to intracellular compartments. In humans, chronic hypophosphatemia results in decreased tissue insulin sensitivity, even in a euglycemic state [5]. Phosphorus infusion to healthy volunteers improves insulin sensitivity under euglycemic conditions [20], and phosphorus supplementation for hypophosphatemic patients with glucose intolerance ameliorates glucose tolerance [21]. Therefore, a reduction in phosphate levels may decrease the peripheral utilization of glucose, thus, leading to the development of insulin resistance, a major determinant of MetS [4].

The strengths of our study include a relatively large, well-characterized, community-based population. In addition, to decrease the influence of diurnal variation in SP levels [22], all phosphorus measurements were conducted under conditions of morning fasting. Furthermore, the available diverse variables, including hs-CRP, an inflammatory marker, were used to minimize confounding in the multivariable-adjusted models. However, the pres- ent study also has some limitations. First, this study had a cross-sectional design, which did not permit the determination of causality. Second, nutritional records were not available, and we could not include phosphorus intake in the analyses. Third, we lacked measurements of urinary phosphorus excretion. Finally, the study population consisted only a section of the Japanese population; therefore, it is uncertain whether these findings can be generalized to other ethnic groups.

In conclusion, lower SP levels were found to be associated with the incidence of MetS only in men. Furthermore, systolic and diastolic BP and TG were negatively correlated with SP levels in men, whereas the inverse correlation was found in women. Our results suggest that there may be gender-related differences in the association of decreased SP levels with MetS and its components.

\section{Acknowledgments}

The authors gratefully acknowledge the contribution of Nobuo Shimosawa in preparing an electronic database of the participants' clinical records. The page charge of this paper is supported by Dr. Manabu Hiraguri, Department of Surgery, Iida Municipal Hospital.

\section{Disclosure Statement}

All authors declare no conflicts of interest.

\section{References}

1 National Cholesterol Education Program (NCEP) Expert Panel on Detection, Evaluation, and Treatment of High Blood Cholesterol in Adults (Adult Treatment Panel III): Third Report of the National Cholesterol Education Program (NCEP) Expert Panel on Detection, Evaluation, and Treatment of High Blood Cholesterol in Adults (Adult Treatment Panel III) final report. Circulation 2002;106:3143-3421.

2 Berndt T, Thomas LF, Craig TA, Sommer S, Li X, Bergstralh EJ, Kumar R: Evidence for a signaling axis by which intestinal phosphate rapidly modulates renal phosphate reabsorption. Proc Natl Acad Sci U S A 2007;104: 11085-11090.

3 Haglin L: Hypophosphataemia: cause of the disturbed metabolism in the metabolic syndrome. Med Hypotheses 2001;56:657-663.

4 Kalaitzidis R, Tsimihodimos V, Bairaktari E, Siamopoulos KC, Elisaf M: Disturbances of phosphate metabolism: another feature of metabolic syndrome. Am J Kidney Dis 2005; 45:851-858.
5 DeFronzo RA, Lang R: Hypophosphatemia and glucose intolerance: evidence for tissue insensitivity to insulin. N Engl J Med 1980; 303:1259-1263.

6 Haap M, Heller E, Thamer C, Tschritter O, Stefan N, Fritsche A: Association of serum phosphate levels with glucose tolerance, insulin sensitivity and insulin secretion in nondiabetic subjects. Eur J Clin Nutr 2006;60: 734-739.

7 Sciacqua A, Perticone M, Cimellaro A, Tassone EJ, Tripepi G, Andreucci M, Sesti G, Perticone F: Multiplicative effect of serum phosphorus levels and insulin resistance on hypertensive vascular stiffness. Thromb Haemost 2016;115:227-229.

8 Gudmundsdottir H, Strand AH, Kjeldsen SE, Hoieggen A, Os I: Serum phosphate, blood pressure, and the metabolic syndrome20 -year follow-up of middle-aged men. J Clin Hypertens (Greenwich) 2008;10:814-821.

9 Lippi G, Montagnana M, Salvagno GL, Targher G, Guidi GC: Relationship between serum phosphate and cardiovascular risk fac- tors in a large cohort of adult outpatients. Diabetes Res Clin Pract 2009;84:e3-e5.

10 Park W, Kim BS, Lee JE, Huh JK, Kim BJ, Sung KC, Kang JH, Lee MH, Park JR, Rhee EJ, Oh KW, Lee WY, Park CY, Park SW, Kim SW: Serum phosphate levels and the risk of cardiovascular disease and metabolic syndrome: a double-edged sword. Diabetes Res Clin Pract 2009;83:119-125.

11 Arai H, Yamamoto A, Matsuzawa Y, Saito Y, Yamada N, Oikawa S, Mabuchi H, Teramoto T, Sasaki J, Nakaya N, Itakura H, Ishikawa Y, Ouchi Y, Horibe H, Kita T; Prevalence of the metabolic syndrome in elderly and middle-aged Japanese: J Clin Gerontol Geriatr 2010;1:42-47.

12 Ishii S, Tanaka T, Akishita M, Ouchi Y, Tuji T, Iijima K: Metabolic syndrome, sarcopenia and role of sex and age: cross-sectional analysis of Kashiwa cohort study. PLoS One 2014;9: e112718.

13 Lee S, Ko Y, Kwak C, Yim ES: Gender differences in metabolic syndrome components among the Korean 66-year-old population with metabolic syndrome. BMC Geriatr 2016;16:27.
Phosphorus Levels and Metabolic Syndrome
Ann Nutr Metab 2017;71:150-156 DOI: $10.1159 / 000480354$ 
14 Haglin L, Lindblad A, Bygren LO: Hypophosphataemia in the metabolic syndrome. Gender differences in body weight and blood glucose. Eur J Clin Nutr 2001;55:493-498.

15 Matsuo S, Imai E, Horio M, Yasuda Y, Tomita K, Nitta K, Yamagata K, Tomino Y, Yokoyama H, Hishida A: Revised equations for estimated GFR from serum creatinine in Japan. Am J Kidney Dis 2009;53:982-992.

16 Onat A, Can G, Kaya H, Hergenc G: "Atherogenic index of plasma” (log10 triglyceride/ high-density lipoprotein-cholesterol) predicts high blood pressure, diabetes, and vascular events. J Clin Lipidol 2010;4:89-98.

17 Onat A, Can G, Ornek E, Sansoy V, Aydin M, Yuksel H: Abdominal obesity with hypertri- glyceridaemia, lipoprotein(a) and apolipoprotein A-I determine marked cardiometabolic risk. Eur J Clin Invest 2013;43:11291139.

18 Onufrak SJ, Bellasi A, Cardarelli F, Vaccarino V, Muntner P, Shaw LJ, Raggi P: Investigation of gender heterogeneity in the associations of serum phosphorus with incident coronary artery disease and all-cause mortality. Am J Epidemiol 2009;169:67-77.

19 Park KS, Chang JW, Kim TY, Kim HW, Lee EK, Kim HS, Yang WS, Kim SB, Park SK, Lee SK, Park JS: Lower concentrations of serum phosphorus within the normal range could be associated with less calcification of the coronary artery in Koreans with normal renal function. Am J Clin Nutr 2011;94:14651470.

20 Khattab M, Abi-Rashed C, Ghattas H, Hlais $\mathrm{S}$, Obeid O: Phosphorus ingestion improves oral glucose tolerance of healthy male subjects: a crossover experiment. Nutr J 2015;14: 112

21 Wittmann I, Nagy J: Effectiveness of phosphate supplementation in glucose intolerant, hypophosphatemic patients. Miner Electrolyte Metab 1997;23:62-63.

22 de Boer IH, Rue TC, Kestenbaum B: Serum phosphorus concentrations in the third $\mathrm{Na}$ tional Health and Nutrition Examination Survey (NHANES III). Am J Kidney Dis 2009; 53:399-407. 